\title{
Negative Predictive Value (NPV)
}

National Cancer Institute ( $\mathrm{NCl})$

\section{Source}

National Cancer Institute (NCI). Negative predictive value.

The likelihood that an individual with a negative test result is truly unaffected and/or does not have the particular gene mutation in question. Also called NPV. 